\title{
The Feasibility and Efficacy of Ablation of Paroxysmal Atrial Fibrillation Using Different Technologies
}

\author{
Samir Rafla ${ }^{1^{*}}$, Amr Kamal' ${ }^{1}$, Mostafa Nawar ${ }^{1}$, Josef Kautzner ${ }^{2}$ \\ ${ }^{1}$ Department of Cardiology, Alexandria University, Egypt \\ ${ }^{2}$ Department of Cardiology, Institute for Clinical and Experimental Medicine (IKEM), Prague, Czech.
}

Received Date: March 11, 2019; Accepted Date: March 20, 2019; Published Date: March 29, 2019

*Corresponding Author: Samir Rafla, Department of Cardiology, Alexandria University, Egypt. Tel: +201001495577; Email:smrafla@yahoo.com

\section{Abstract}

This analysis assesses the efficacy of ablation of paroxysmal atrial fibrillation (PAF). Methods: We studied 150 patients (pts) (86 males and 64 females) having a mean age of 51.3 yrs. (54 > 50, 96 below 50 yrs.), who suffered from symptomatic drug refractory paroxysmal AF. Cardiac MSCT (Multi Slice Computed Tomography) image integration to the 3D electroanatomic LA map was used in 106 pts $(70.6 \%$, however all of them underwent intracardiac echo guided imaging during the ablation procedure. $40 \mathrm{pts}$ underwent manual radio frequency (RF) ablation using CARTO, 40 pts underwent ablation using NavX system, 70 pts underwent robotic ablation using Sansui system. Results: 34 patients (22.6\%) developed early recurrence of AF after an initial blanking period of 3 months. The incidence of recurrence of AF in males was $13 \%(11 / 86), 14 \%$ in females $(9 / 64), P=N S$. ECG during follow up: Atrial Tachycardia 4, PAF 2, A. flutter 2. Complications: air embolism zero, cardiac tamponade zero, trivial pericardial effusion 1 , groin hematoma 5\%, pulmonary vein stenosis $>50 \%$ zero. There was no difference between males and females in success of ablation or complications. Those below age 50 and above 50 were not different in incidence of maintenance of SR or complications. In all, the mean age was 53.6 years in successful group and 58 years in failure group, with no significant differences between both groups. Conclusions: The success rate for the robotic group was $92.4 \%$. The manually treated group (Carto and NavX) has a success rate of $88.5 \%$.
Keywords: Ablation; Paroxysmal Atrial Fibrillation; Pulmonary vein isolation; Robotic ablation

The aim of this study was to evaluate the feasibility of catheter ablation in patients with paroxysmal atrial fibrillation using different technologies. The effect on procedural efficacy and success rate was analyzed.

\section{Introduction}

Catheter ablation (CA) is an effective therapy for the treatment of symptomatic drug-refractory paroxysmal atrial fibrillation (AF) [1-6]. Pappone et al. [2] compared the efficacy and safety of circumferential PV ablation with antiarrhythmic drug treatment in a large number of patients with long-term follow-up, and showed that ablation therapy significantly improved the morbidity and mortality of AF patients. Three randomized trials in patients with paroxysmal AF demonstrated that catheter ablation was superior to antiarrhythmic therapy in the prevention of recurrent AF [7-8]. Also in the Catheter Ablation for the Cure of Atrial Fibrillation study (CACAF), catheter ablation plus antiarrhythmic drugs was compared with antiarrhythmic drugs alone, and addition of ablation therapy was superior [9]. This was further confirmed in the Catheter Ablation versus Antiarrhythmic Drugs for Atrial Fibrillation [4] and in the Circumferential Pulmonary Vein Ablation versus Antiarrhythmic Drug Therapy in Paroxysmal Atrial Fibrillation (APAF) trials [10-12], which showed that it is unquestionable that ablation therapy is more effective than antiarrhythmic drugs, at least in young mostly healthy patients 
and when performed by highly skilled practitioners. Catheter ablation of $\mathrm{AF}$ is also appropriate in selected symptomatic patients with heart failure and/or reduced ejection fraction.

Current Indications for Catheter Ablation of Atrial Fibrillation: The ACC/AHA/ESC/HRS 2012 Guidelines for the Management of Patients with Atrial Fibrillation, considers ablation Class I indication (level of evidence A). Ablation Approaches Targeting the Pulmonary Veins (PV): Although ablation strategies, which target the PVs, remain the cornerstone of AF ablation procedures for both paroxysmal and persistent AF, continued efforts are underway to identify additive strategies to improve outcome. Instead of targeting specific muscular connections between the PVs and the left atrium (LA), the entire PV antrum was ablated along its border and pulmonary vein stenosis became an increasingly rare complication. Studies comparing the efficacy of segmental ostial ablation to a wide, circumferential antral isolation clearly demonstrated a benefit to the latter approach with better success rates of AF ablation [13]. At the present time, Pulmonary vein antral isolation (PVAI) remains the cornerstone of the most widely adopted approach to catheter ablation of AF.

The goal of ablation is to eliminate all Pulmonary vein potentials (PVPs) within and around the PV antra by ablation. As the circular catheter is moved from one segment of the LAantral interface to the next, ablation is performed at the poles that demonstrate PVPs.

Ablation Approaches Not Targeting the Pulmonary Veins: Non-PV triggers initiating AF can be identified in up to one third of unselected patients referred for catheter ablation for paroxysmal AF [14].

Alternate Ablation Energy Sources: Although RF energy is most commonly employed for catheter ablation of AF, a number of alternative catheter ablation systems that utilize different ablative energy sources have been developed and currently are being evaluated in clinical trials. These include cryoablation, ultrasound ablation, and laser ablation [15-17].

\section{Methods}

We studied 150 patients (pts) (86 males and 64 females) having a mean age of 51.3 yrs $(54>50,96$ below 50 yrs), who suffered from symptomatic drug refractory paroxysmal AF. Work was done in IKEM center in Prague from 2008 to 2010.

\section{Patients were subjected to the following:}

- Full History Taking \& Clinical Examination,

- Baseline 12- Lead Electrocardiogram (ECG).

- Routine Laboratory Investigations (blood count, kidney and liver function, blood sugar, HbA1c (glycated hemoglobin).
- Cardiac Imaging Modalities: Chest X ray Examination, Transthoracic Echocardiography (TTE), Transesophageal Echocardiography (TEE), Cardiac Multislice Computed Tomography (MSCT), Intracardiac Echocardiography (ICE),

- Preprocedural Management: Informed consent, Preprocedural anticoagulation, Preprocedural antiarrhythmic drugs.

- Procedural management: Vascular access, Procedural sedation, Procedural anticoagulation, Double transseptal puncture, Catheters positioning.

- Three dimensional electroanatomic mapping: The EnSite NavX® system (Endocardial Solutions, St. Jude Medical, Inc.). The CARTO mapping system (Biosense, Diamond Bar, CA, USA)

- Radiofrequency Catheter Ablation: Robotic Catheter Navigation System (Sensei System, Hansen Medical, Inc.), Manual Catheter Ablation.

- Post procedural management \& follow up patients were followed up regularly at the outpatient arrhythmia Clinic at 3, 6, 9 and 12 months, as well as at any time for any possible attacks of arrhythmic recurrences.

\section{Patients were followed up as regards:}

Clinical symptoms, Standard 12- lead Electrocardiogram (ECG), in hospital Telemetry, 7- Day Holter Monitoring, Outpatient Mobile Telemetry with Loop Recording the patients were divided into three groups:

- Group C (Carto): Forty patients with paroxysmal atrial fibrillation who underwent ablation using CARTO technology and manual ablation.

- Group N (NavX): Forty patients with paroxysmal atrial fibrillation who underwent ablation using NavX technology and manual ablation.

- Group R (Robotic): Seventy patients with paroxysmal atrial fibrillation who underwent ablation using NavX technology with use of robotic catheter navigation system (Sensei System). Integration of CT Image into CARTO Mapping System: CT image fusion with 3D Carto map was done to most of the patients; the CT image was imported into the EAM system using special software (Cartomerge $^{\mathrm{TM}}$, Biosense Webster, Inc., Diamond Bar, CA, USA) Integration of CT Image into EnSite NavX Mapping System: The contrast enhanced CT image in standard DICOM format was imported into the mapping system using the EnSite System software tools for digital image fusion in the same way. 


\section{Abbreviations}

\begin{tabular}{|l|l|}
\hline TTE & Transthoracic Echocardiography \\
\hline AAD & Antiarrhythmic drugs. \\
\hline ACD & Anticoagulation Drug. \\
\hline CARTO & Cardiac mapping system / electromagnetic. \\
\hline CFAEs & Complex fractionated atrial electrograms \\
\hline CPVA & Circumferential pulmonary vein ablation. \\
\hline CT & Computed Tomography. \\
\hline CTI & Cavotricuspid isthmus. \\
\hline CVS & Cerebro-vascular stroke. \\
\hline EAM & Electroanatomic map. \\
\hline ICE & Intracardiac echocardiography. \\
\hline MSCT & Cardiac Multislice Computed Tomography \\
\hline PVAI & Pulmonary vein antral isolation \\
\hline PVI & Pulmonary vein isolation. \\
\hline
\end{tabular}

\section{Ablation Procedure}

Ablation was done in all patients using the open irrigation ablation catheter in the power controlled mode either manually or after mounting on Artisan catheter for remote robotic catheter navigation system. The end point of the ablation was the disconnection between the PV and LA (disappearance of pulmonary vein potentials), and noninducibility of AF/AFL. Periprocedural Anticoagulation during AF Catheter Ablation: After the procedure, heparin infusion is discontinued. Warfarin therapy is restarted in all patients either the same evening of the ablation procedure or next morning. In the initial period, LMWH (e.g., Enoxaparin at a dosage of 0.5-1.0 $\mathrm{mg} / \mathrm{kg}$ twice a day) is often given as bridging therapy by starting 3-4 hours after the ablation or alternatively heparin is administered intravenously until the day after the procedure, starting about 3 hours after sheath removal at a rate of 1000 $\mathrm{IU} / \mathrm{h}$. Thereafter, LMWH is administrated until the INR is $\geq 2$. Once the therapeutic INR is achieved, LMWH is stopped, whereas warfarin is continued for at least 3 months. The anticoagulation strategy after the initial 3 months varies according to patient and procedure related factors and for most patients with a CHADS2 score of $\geq 2$ to continue long-term warfarin treatment with a targeted INR of $2-3$ is usually needed.

Cardiac MSCT image integration to the 3D electroanatomic LA map was used in 106 pts (70.6\%, however all of them underwent intracardiac echo guided imaging during the ablation procedure. 40 pts underwent manual RF ablation using CARTO, 40 pts underwent ablation using NavX system, 70 pts underwent robotic ablation using Sensui system. Pulmonary vein isolation was done to all pts using either pulmonary vein (PV) antral isolation in $116(77.3 \%)$ or circumferential pulmonary vein ablation in 34 pts $(22.7 \%)$. All pts were followed at 3,6,9, and 12 months.

\section{Statistical Analysis of the Data}

Data were fed to the computer and analyzed using IBM SPSS software package version 20.0. Qualitative data were described using number and percentage. Quantitative data were described using mean and standard deviation. Comparison between different groups regarding categorical variables was tested using Chi-square test. When more than $20 \%$ of the cells have expected count less than 5, correction for chi-square was conducted using Fisher's exact test. Correlations between two quantitative variables were assessed using Pearson coefficient. Significance of the obtained results was judged at the 5\% level. Data was presented as Median (Min. - Max.) for abnormally distributed data or Mean \pm SD. for normally distributed data.

\section{Results}

The present study included one hundred and fifty patients suffered from symptomatic drug refractory paroxysmal atrial fibrillation, after assessing all the patients as regards demographic data, clinical data, procedural data and follow up data, analysis of the results was done using version 17 of the SPSS statistics software.

\section{The patients were divided into three groups as mentioned} in methods:

- Group C (Carto): Forty patients with paroxysmal atrial fibrillation who underwent ablation using CARTO technology and manual ablation.

- Group N (NavX): Forty patients with paroxysmal atrial fibrillation who underwent ablation using NavX technology and manual ablation.

- Group R (Robotic): Seventy patients with paroxysmal atrial fibrillation who underwent ablation using NavX technology with use of robotic catheter navigation system (Sensei System).

Comparison between the three groups was conducted and analyzed in the study results, moreover, comparison between manually treated patients group (Carto and NavX) versus Robotic group was also done. Univariate analysis of variables at each group of the three groups was done and multivariate analysis and predictors of success were statistically calculated and analyzed. 


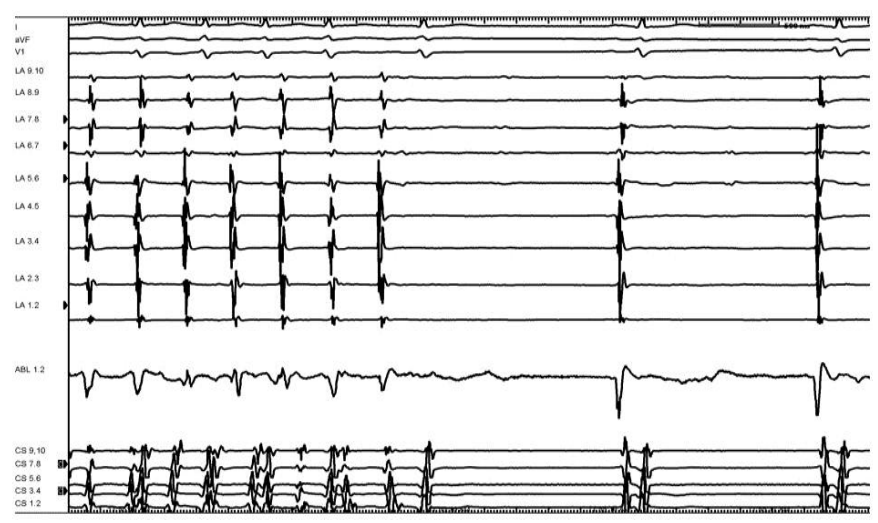

Figure 1: Termination of AF during ablation.

Demographic Data: The mean age was significantly lower in Robot group (46.1 \pm 11.0 years) compared to Carto group (54.5 \pm 8.0 years $)$ and group $\mathrm{NavX}(53.7 \pm 12.2$ years $), \mathrm{P}<0.001$. The percentage of male patients was significantly higher in Carto group $(70 \%)$ and NavX group (65\%) compared to Robot group (45.7\%), $\mathrm{P}<0.05$.

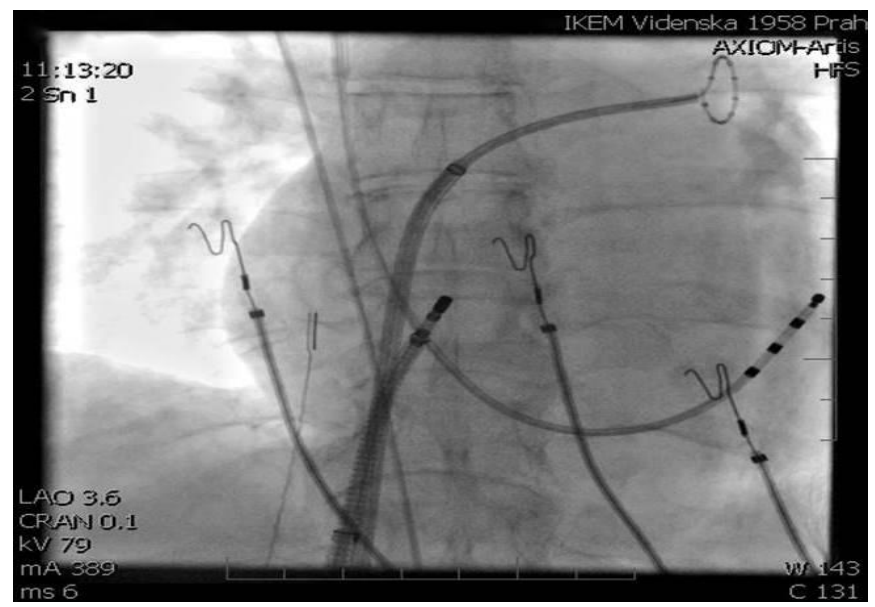

Figure 2: Fluoroscopic PA view with Lasso catheter in transseptal sheath placed at LSPV and quadripolar CS catheter in place with Artisan robotic catheter navigating through the Interatrial septum(IAS). Note Intracardiac echocardiography) ICE catheter in RA (abbreviations in text).

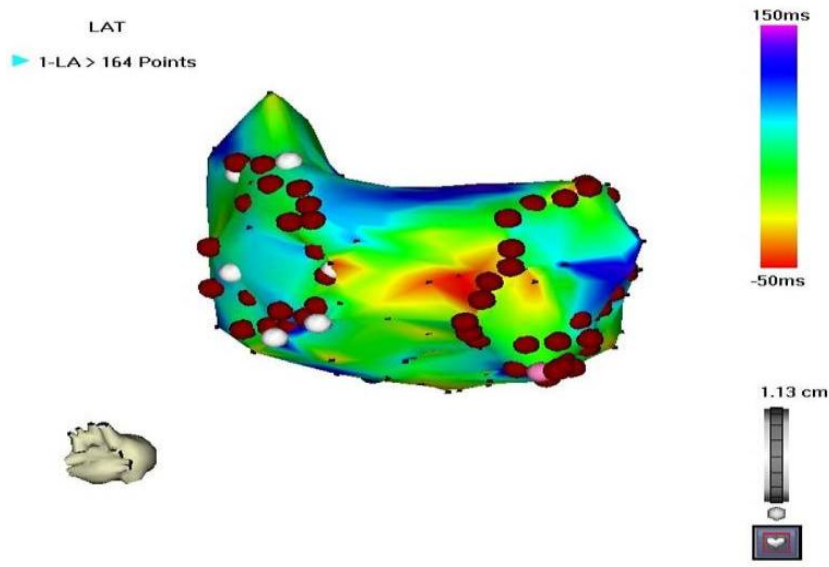

Figure 3: Activation Carto map. 


\begin{tabular}{|c|c|c|c|c|c|c|c|c|}
\hline & \multicolumn{2}{|c|}{$\begin{array}{c}\text { Group C } \\
(n=40)\end{array}$} & \multicolumn{2}{|c|}{$\begin{array}{c}\text { Group N } \\
(n=40)\end{array}$} & \multicolumn{2}{|c|}{$\begin{array}{c}\text { Group R } \\
(\mathbf{n}=70)\end{array}$} & $\mathrm{X} 2$ & $\mathbf{P}$ \\
\hline & No & $\%$ & No & $\%$ & No & $\%$ & \multirow{3}{*}{41.04} & \multirow{3}{*}{$0.000 *$} \\
\hline PV triggers strategy PVAI & 20 & 50 & 26 & 65 & 70 & 100 & & \\
\hline CPVA & 20 & 50 & 14 & 35 & 0 & 0 & & \\
\hline $\begin{array}{c}\text { Non PV triggers strategy Not } \\
\text { done }\end{array}$ & 18 & 45 & 24 & 60 & 60 & 85.7 & \multirow{4}{*}{42.24} & \multirow{4}{*}{$0.000 *$} \\
\hline LA posterior wall ablation & 16 & 40 & 12 & 30 & 0 & 0 & & \\
\hline $\begin{array}{c}\text { Posterior wall ablation+ } \\
\text { CFAEs }\end{array}$ & 4 & 10 & 2 & 5 & 0 & 0 & & \\
\hline CFAEs ablation & 2 & 5 & 2 & 5 & 10 & 14.3 & & \\
\hline $\begin{array}{l}\text { PVA } \\
\text { CFAEs } \\
\text { PVAI }\end{array}$ & $\begin{array}{l}\text { Circun } \\
\text { Compl } \\
\text { Pulmor }\end{array}$ & $\begin{array}{l}\text { tiona } \\
\text { in a }\end{array}$ & $\begin{array}{l}\text { al ele } \\
\text { latior }\end{array}$ & ion. & & & & \\
\hline
\end{tabular}

Table 1: Comparison between the three groups as regards ablation strategy.

\begin{tabular}{|c|c|c|c|c|c|c|c|c|c|}
\hline & Groups & $\begin{array}{c}\text { No of } \\
\text { patients }\end{array}$ & Min & Max & Mean & SD & $\mathbf{F}$ & $\mathbf{P}$ & LSD \\
\hline \multirow{3}{*}{ Procedural time } & $\mathrm{C}$ & 40 & 170 & 230 & 206.5 & 18.4 & \multirow{3}{*}{239.2} & \multirow{3}{*}{$0.000 *$} & R vs C,N \\
\hline & $\mathrm{N}$ & 40 & 170 & 240 & 197.5 & 19.9 & & & \multirow{2}{*}{$\mathrm{C}$ vs $\mathrm{N}$} \\
\hline & $\mathrm{R}$ & 70 & 120 & 165 & 146.4 & 10.8 & & & \\
\hline \multirow{3}{*}{$\begin{array}{c}\text { Total fluoroscopy } \\
\text { time }\end{array}$} & $\mathrm{C}$ & 40 & 13.7 & 25.1 & 19.3 & 3.2 & \multirow{3}{*}{493} & \multirow{3}{*}{$0.000 *$} & $\mathrm{R}$ vs $\mathrm{C}, \mathrm{N}$ \\
\hline & $\mathrm{N}$ & 40 & 17 & 25.5 & 20.5 & 2.9 & & & \\
\hline & $\mathrm{R}$ & 70 & 3.5 & 10 & 6.9 & 1.9 & & & C vs $N$ \\
\hline \multirow{3}{*}{$\begin{array}{c}\text { Total fluoroscopy } \\
\text { dose }\end{array}$} & $\mathrm{C}$ & 40 & 1368 & 3422 & 2226.3 & 634.9 & \multirow{3}{*}{284.8} & \multirow{3}{*}{$0.000 *$} & \multirow{3}{*}{ R vs C,N } \\
\hline & $\mathrm{N}$ & 40 & 1760 & 3176 & 2288 & 515.5 & & & \\
\hline & $\mathrm{R}$ & 70 & 210 & 995 & 552.7 & 194.1 & & & \\
\hline \multirow{3}{*}{ 3D EAM time } & $\mathrm{C}$ & 40 & 18 & 25 & 22.5 & 2.1 & \multirow{3}{*}{4.79} & \multirow{3}{*}{$0.010^{*}$} & \multirow{3}{*}{ C vs R,N } \\
\hline & $\mathrm{N}$ & 40 & 18 & 25 & 21.6 & 2 & & & \\
\hline & $\mathrm{R}$ & 70 & 17 & 25 & 21.3 & 1.9 & & & \\
\hline \multirow{3}{*}{ Total PVI time } & $\mathrm{C}$ & 40 & 58 & 105 & 83.8 & 11.8 & \multirow{3}{*}{100.4} & \multirow{3}{*}{$0.000^{*}$} & \multirow{3}{*}{$\mathrm{R}$ vs $\mathrm{C}, \mathrm{N}$} \\
\hline & $\mathrm{N}$ & 40 & 61 & 109 & 83.2 & 12.8 & & & \\
\hline & $\mathrm{R}$ & 70 & 50 & 70 & 61.9 & 4.3 & & & \\
\hline \multirow{3}{*}{ Total ablation time } & $\mathrm{C}$ & 40 & 1200 & 4555 & 2619.9 & 881.7 & \multirow{3}{*}{89.64} & \multirow{3}{*}{$0.000^{*}$} & $\mathrm{R}$ vs $\mathrm{C}, \mathrm{N}$ \\
\hline & $\mathrm{N}$ & 40 & 1870 & 3000 & 2373.5 & 364 & & & \multirow{2}{*}{$\mathrm{C}$ vs $\mathrm{N}$} \\
\hline & $\mathrm{R}$ & 70 & 750 & 2000 & 1323.1 & 355.2 & & & \\
\hline & & & VI: $\mathrm{Pu}$ & nary & isolation. & & & & \\
\hline
\end{tabular}

Table 2: Comparison between the three groups as regards overall procedures.

\begin{tabular}{|c|c|c|c|c|c|c|c|c|}
\hline \multirow{2}{*}{ Complications } & \multicolumn{2}{|c|}{$\begin{array}{c}\text { Group C } \\
(n=40)\end{array}$} & \multicolumn{2}{|c|}{$\begin{array}{c}\text { Group N } \\
(n=40)\end{array}$} & \multicolumn{2}{|c|}{$\begin{array}{c}\text { Group R } \\
(\mathbf{n}=70)\end{array}$} & \multirow{2}{*}{$\mathbf{X} 2$} & \multirow[t]{2}{*}{$\mathbf{P}$} \\
\hline & No & $\%$ & No & $\%$ & No & $\%$ & & \\
\hline No complications & 37 & 92.5 & 37 & 92.5 & 66 & 94.4 & 0.218 & 0.896 \\
\hline Trivial pericardial effusion & 1 & 2.5 & 1 & 2.5 & 0 & 0 & 1.773 & 0.411 \\
\hline
\end{tabular}




\begin{tabular}{|c|c|c|c|c|c|c|c|c|}
\hline Cardiac tamponade & 0 & 0 & 0 & 0 & 0 & 0 & 0 & 1 \\
\hline Air embolism & 0 & 0 & 0 & 0 & 1 & 1.4 & 1.15 & 0.168 \\
\hline Small groin hematoma & 2 & 5 & 2 & 5 & 3 & 4.2 & 0.24 & 0.345 \\
\hline Thromboembolism & 0 & 0 & 0 & 0 & 0 & 0 & 0 & 1 \\
\hline
\end{tabular}

Table 3: Comparison between the three groups as regards complications.

\begin{tabular}{|c|c|c|c|c|c|c|}
\hline Long term & manu & $n=40)$ & Robot & $n=35$ ) & $\mathbf{X} 2$ & $\mathbf{P}$ \\
\hline Good( without AAD) & 62 & 77.5 & 60 & 85.7 & \multirow{4}{*}{2.578} & \multirow{4}{*}{0.276} \\
\hline Average(with AAD) & 4 & 5 & 4 & 5.7 & & \\
\hline $\begin{array}{c}\text { Comprehensive (total) } \\
\text { success }\end{array}$ & 66 & 82.5 & 64 & 91.4 & & \\
\hline Failure & 14 & 17.5 & 6 & 8.6 & & \\
\hline
\end{tabular}

Table 4: Comparison between manual and robotic groups as regards procedural efficacy and success rate.

Duration of Symptoms: The mean duration of symptoms was $4.1 \pm 2.0$ years in Carto group, $3.2 \pm 1.4$ years in NavX group and $3.5 \pm 1.9$ years in Robot group. There was no significant difference between the three groups, $\mathrm{P}=0.063$. Duration of AF: Duration of AF was significantly longer in Carto group (3.7 \pm 1.3 years) compared to NavX group ( $2.6 \pm$ 0.8 years) and Robot group ( $2.4 \pm 0.9$ years).Coronary artery disease was present in $20 \%$ of patients of Carto group, $25 \%$ of patients of NavX group and $28.6 \%$ of patients of Robot group. There was no significant difference between the three groups.

Systolic heart failure was present in $10 \%$ of patients of Carto group, 5\% of patients of NavX group and $11.4 \%$ of patients of Robot group. There was no significant difference between the three groups. Associated Typical Atrial Flutter:

Associated atrial flutter was present in $25 \%$ of Carto group, $15 \%$ of NavX group and $8.6 \%$ of Robot group, with no significant difference between the three groups.

CHADS2 Score and History of Thromboembolic Stroke: The percentage of patients with CHADS2 of value > 1 was significantly more in Carto group (75\%) compared to NavX group (50\%) and Robot group (42.9\%). The percentage of patients with past history of cerebro-vascular stroke (CVS) as a complication of AF was significantly higher in Carto group (15\%) compared to NavX group (0\%) and Robot group $(2.9 \%)$.

Preprocedural Anti-Arrhythmic Drug History: Propafenone, amiodarone, flecainide were the most common AADs used in the three groups with no significant difference. Flecainide was significantly used more in NavX group (15\%) compared to Carto group (5\%), while sotalol was significantly used more in Carto group (15\%) compared to NavX group $(0 \%)$. The AADs were not effective in nearly total patients of the three groups.MSCT Image Integration: CARTO Merge was used in 70\% of patients of Carto group and MSCT image integration was used in $70 \%$ of NavX group and $71.4 \%$ of Robot group.
Previous EP Procedures: Most of the patients in the three groups had no previous EP procedures $(60 \%$ in Carto group, $80 \%$ in NavX group and $77.1 \%$ in Robot group). CTI ablation was done to $20 \%$ of patients of Carto group and NavX group and $11.4 \%$ of patients of Robot group. The percentage of patients with DDDR pacemaker was significantly higher in Carto group (15\%) compared to the other two groups $(0 \%)$. Atrio-Ventricular Nodal Reentrant Tachycardia (AVNRT) ablation and 2D ICD were done to small numbers in the three groups.

Preprocedural Anticoagulation Drug (ACD) History: There was no significant difference between the three groups as regards the duration of ACD treatment. 3D Electroanatomic Mapping: CARTO 3D mapping system was used in all patients of Carto group and EnSite NavX 3 D mapping system was used in all patients of the other two groups.

ECG during follow up: Atrial Tachycardia 4, PAF 2, A. flutter 2. Complications rate: None in $92,5 \%$, air embolism zero, cardiac tamponade zero, trivial pericardial effusion 1, groin hematoma $5 \%$, pulmonary vein stenosis $>50 \%$ zero.

There was no difference between males and females in success of ablation or complications. Those below age 50 and above 50 were not different in incidence of maintenance of SR or complications.

In the all patients, the mean age was $53.6 \pm 8.2$ years in successful group and $58 \pm 6.2$ years in failure group, with no significant differences between both groups.

Comparison between successful and failed cases in Robotic ( $\mathrm{R})$ group: The mean age was $(45.2 \pm 10.8$ years) in successful group and (55.7 \pm 7.7 years) in failure group. It was significantly higher in failure group.

\section{Discussion}

Catheter ablation of $\mathrm{AF}$ is now a realistic therapeutic option for patients with paroxysmal AF [18]. In this study, one 
hundred and fifty patients were enrolled for catheter ablation of symptomatic paroxysmal AF who had failed at least one antiarrhythmic drug.

Pulmonary vein isolation is the cornerstone in treatment for paroxysmal AF. Electrical isolation of all pulmonary veins is the endpoint of ablation, and this objective measure of pulmonary vein disconnection is easy to confirm and published results in maintenance of sinus rhythm in between $60 \%$ and $85 \%$ of patients $[\mathbf{1 9}, \mathbf{2 0}]$, the difficulty is how to improve on this.

The role of additional substrate modification in paroxysmal AF is controversial and the question that is not yet fully answered is how to determine which patients require substrate modification in addition to pulmonary vein isolation during the index procedure.

The aim of our study is to evaluate the feasibility of paroxysmal atrial fibrillation ablation using different technologies and its effect on terms of procedural efficacy and success rate as well as evaluation of different approaches and strategies during catheter ablation of atrial fibrillation aiming at reaching a multivariate analysis of predictors of success.

Our study describes the clinical use of different technologies for catheter ablation of paroxysmal AF using two different 3D-electroanatomical mapping systems (Carto and NavX), real time intracardiac echocardiography (ICE) imaging and multislice cardiac CT imaging, as well as novel robotic catheter navigation system. In our study, a multivariate analysis of predictors of success for patients with paroxysmal AF who underwent ablation using Carto 3D EAM technology and manual ablation was done and different variables were evaluated as regards their significance as predictors of success.

The significant predictors of success in Carto group were PV antral isolation as the used method of eliminating PV triggers, PV antrum as a target PV ablation site, early recurrence during blanking period, rhythm outcome, baseline $\mathrm{ECG}$, duration of AF and additional ablation line (roof line) in order, while other predictors were not significant.

Our study concluded that age was not a significant predictor of success in Carto group and the success of catheter ablation of paroxysmal AF did not depend on age of the patient, and catheter ablation of AF is safe procedure in all age groups, a similar conclusion was reported by Bhargava et al. [21] who stated that PVI is a safe and effective treatment for patients with drug-refractory symptomatic AF, and its benefits extend to all age groups. However they added that risk of procedural complications, especially thromboembolic events, appears to be higher in the elderly age group [21].

Zado et al. [22] showed that elderly patients with AF undergoing catheter ablation therapy have a higher incidence of hypertension/structural heart disease.
Moreover, to achieve a similar level of AF control, it appears to be no increased risk from the ablation procedure, but elderly patients are more likely to remain on antiarrhythmic drugs [22].

In our study multislice cardiac CT image integration into the 3D electroanatomical map either into Carto using Carto Merge software was used in $75 \%$ of successful group and $50 \%$ of failure group with no significant difference. According to our data, MSCT Image integration to guide catheter ablation for AF did not significantly improve the clinical outcome in Carto group.

Our observations suggest also that achieving electrical PVI is the critical determinant of procedural success rather than the mapping tools used to achieve it, this may be also attributed to the use of ICE for all patients during construction of the three dimensional electroanatomical left atrial map and the experience of the operator that may allow an accurate reconstruction of chamber geometry without the use of integrated MSCT image into the 3D electroanatomic map.

The same conclusion was agreed by Kistler et al. [23], who concluded that achieving electrical PVI is the critical determinant of procedural success rather than the mapping tools used to achieve this goal.

\section{Conclusion}

As regards the primary and secondary procedural efficacy endpoint and the comprehensive success rate for the three groups; the robotic group showed a constant comprehensive success rate over the follow up period of $92.4 \%$ with no recurrences associated with a trend towards withdrawal of AAD. The manually treated group (Carto and NavX) has a comprehensive success rate of $88.5 \%$.

\section{Acknowledgment}

- The authors like to thank all the doctors, technicians and nurses of IKEM Institute hospital for their expertise and help.

- The authors have no conflict of interest.

- The authors did not receive funds from any agent.

\section{References}

1. Jais P, Cauchemez B, Macle L, Daoud E, Khairy P, et al. (2008) Catheter ablation versus antiarrhythmic drugs for atrial fibrillation: the A4 study. Circulation 118: 24982505.

2. Pappone C, Augello G, Sala S, Gugliotta F, Vicedomini $\mathrm{G}$, et al. (2006) A randomized trial of circumferential pulmonary vein ablation versus antiarrhythmic drug therapy in paroxysmal atrial fibrillation: the APAF study. J Am Coll Cardiol 48: 2340-2347. 
3. Camm AJ, Lip GY, De Caterina R, Savelieva I, Atar D, et al. (2012) Focused update of the ESC guidelines for the management of atrial fibrillation: an update of the 2010 ESC guidelines for the management of atrial fibrillation. Eur Heart J 33: 2719-2747.

4. Fuster V, Ryde'n LE, Cannom DS, Crijns HJ, Curtis AB, et al. (2011) 2011 ACCF/AHA/HRS focused updates incorporated into the ACC/AHA/ESC 2006 guidelines for the management of patients with atrial fibrillation. J Am Coll Cardiol 57: e101-198.

5. Pappone C, Rosanio S, Augello G, Gallus G, Vicedomini G, Mazzone,et al. (2003) Mortality, morbidity, and quality of life after circumferential pulmonary vein ablation for atrial fibrillation: outcomes from a controlled nonrandomized long-term study. J Am Coll Cardiol 42: 185-197.

6. Jais P, Cauchemez B, MacLe L, Daoud E, Waldo AL, et al. (2006) Atrial fibrillation ablation vs antiarrhythmic drugs: A multicenter randomized trial. Heart Rhythm 3: S1-S460.

7. Wazni OM, Marrouche NF, Martin DO, Verma A, Bhargava M, et al. (2005) Radiofrequency ablation vs. antiarrhythmic drugs as first-line treatment of symptomatic atrial fibrillation: a randomized trial. JAMA 293: 2634-2640.

8. Oral H, Chugh A, Ozaydin M, Good E, Fortino J, et al. (2006) Risk of thromboembolic events after percutaneous left atrial radiofrequency ablation of atrial fibrillation. Circulation 114: 759-765.

9. Stabile G, Bertaglia E, Senatore G, De Simone A, Zoppo F, et al. (2006) Catheter ablation treatment in patients with drug-refractory atrial fibrillation: a prospective, multicentre, randomized, controlled study (Catheter Ablation For The Cure Of Atrial Fibrillation Study). Eur Heart J 27: 216-221.

10. Callans DJ, Gerstenfeld EP, Dixit S, Zado E, Vanderhoff M, et al. (2004) Efficacy of repeat pulmonary vein isolation procedures in patients with recurrent atrial fibrillation. J Cardiovasc Electrophysiol 15: 1050-1055.

11. Arentz T, von RJ, Blum T, Stockinger J, Burkle G, et al. (2003) Feasibility and safety of pulmonary vein isolation using a new mapping and navigation system in patients with refractory atrial fibrillation. Circulation 108: 24842490.

12. Yamada T, Murakami $Y$, Okada $T$, Okamoto M, Shimizu $\mathrm{T}$, et al. (2006) Electrophysiological pulmonary vein antrum isolation with a multielectrode basket catheter is feasible and effective for curing paroxysmal atrial fibrillation: efficacy of minimally extensive pulmonary vein isolation. Heart Rhythm 3: 377-384.

13. Oral H, Scharf C, Chugh A, Hall B, Cheung $P$, et al. (2003) Catheter ablation for paroxysmal atrial fibrillation: segmental pulmonary vein ostial ablation vs left atrial ablation. Circulation 108: 2355-2360.

14. Shah D, Haissaguerre M, Jais P, Hocini M (2003) Nonpulmonary vein foci: do they exist? Pacing Clin Electrophysiol 26: 1631-1635.

15. Garan A, Al-Ahmad A, Mihalik T, Cartier C, Capuano L, et al. (2006) Cryoablation of the pulmonary veins using a novel balloon catheter. J Interv Card Electrophysiol 15: 79-81.

16. Themistoclakis S, Wazni OM, Saliba W, Schweikert RA, Bonso A, et al. (2006) Endoscopic fiberoptic assessment of balloon occlusion of the pulmonary vein ostium in humans: comparison with phased-array intracardiac echocardiography. Heart Rhythm 3: 44-49.

17. Mont L1, Bisbal F, Hernández-Madrid A, PérezCastellano N, Viñolas X, et al. (2014) Catheter Ablation vs. Antiarrhythmic Drug Treatment of Persistent Atrial Fibrillation. A Multicentre, Randomized, Controlled Trial (SARA Study). Eur Heart J 35: 501-507.

18. Natale A, Raviele A, Arentz T, Calkins H, Chen SA, et al. (2007) Venice Chart International Consensus Document on Atrial Fibrillation Ablation. J Cardiovasc Electrophysiol 18: 560-580.

19. Oral H, Chugh A, Ozaydin M, Good E, Fortino J, et al. (2006) Risk of thromboembolic events after percutaneous left atrial radiofrequency ablation of atrial fibrillation. Circulation August 114: 75-765.

20. Haissaguerre M, Hocini M, Sanders $P$, Sacher F, Rotter $\mathrm{M}$, et al. (2005) Catheter ablation of long-lasting persistent atrial fibrillation: clinical outcome and mechanisms of subsequent arrhythmias. J Cardiovasc Electrophysiol 16: 1138-1147.

21. Bhargava M, Marrouche NF, Martin DO, Schweikert RA, Saliba W, et al. (2004) Impact of age on the outcome of pulmonary vein isolation for atrial fibrillation using circular mapping technique and cooled-tip ablation catheter. J Cardiovasc Electrophysiol 15: 8-13.

22. Zado E, Callans DJ, Riley M, Hutchinson M, Garcia F, et al. (2008) Long-term clinical efficacy and risk of catheter ablation for atrial fibrillation in the elderly. J Cardiovasc Electrophysiol 19: 621-626.

23. Kistler PM, Rajappan K, Harris S, Earley MJ, Richmond L, et al. (2008) The impact of image integration on catheter ablation of atrial fibrillation using electroanatomic mapping: a prospective randomized study. Eur Heart J 29: 3029-3036.

Citation: Rafla S, Kamal A, Nawar M, Kautzner (2019) The Feasibility and Efficacy of Ablation of Paroxysmal Atrial Fibrillation Using Different Technologies. Int Jr Cardiac Scie and Res: IJCSAR-109. 